\title{
THE CONSTRAINED COEFFICIENT PROBLEM FOR TYPICALLY REAL FUNCTIONS $\left({ }^{1}\right)$
}

BY

GEORGE B. LEEMAN, JR.

ABSTRACT. Let $-2 \leq c \leq 2$. In this paper we find the precise upper and lower bounds on the $n$th Taylor coefficient $a_{n}$ of functions $f(z)=z+c z^{2}+$ $\Sigma_{k=3}^{\infty} a_{k} z^{k}$ typically real in the unit disk for $n=3,4, \ldots$ In addition all the extremal functions are identified.

Let $|c| \leq 2$, and denote by $S(c)$ the collection of all functions $f(z)=z+$ $c z^{2}+\sum_{k=3}^{\infty} a_{k} z^{k}$ analytic and univalent in the unit disk $D=\{z|| z \mid<1\}$. This class has been studied by Gronwall [9], [10], Nevanlinna [15], Lebeder and Milin [13], Goluzin [7], and Jenkins [11]. More recently Jenkins [1, pp. 159-174] solved the problem of maximizing $\left|a_{n}\right|$, where $f(z)=z+c z^{2}+\sum_{k=3}^{\infty} a_{k} z^{k} \in S(c)$, for the case $n=3$. This problem has not been solved for any $n \geq 4$.

The purpose of this paper is to give a complete solution to the analogous constrained coefficient problem for a much simpler class of functions, namely, the typically real functions.

Definition 1. A function $f(z)=z+\sum_{k=2}^{\infty} a_{k} z^{k}$ analytic in the unit disk $D$ is said to be typically real provided $f(z)$ is real if and only if $z$ is real. The class of typically real functions will be denoted by $T$, and for each $c,-2 \leq c \leq 2$, we call $T(c)$ the collection of all functions $f(z)=z+c z^{2}+\sum_{k=3}^{\infty} a_{k} z^{k} \in \bar{T}$.

Rogosinski [18], [19] introduced the class $T$ and established many of its important properties. He showed that if $f(z)=z+\sum_{k=2}^{\infty} a_{k} z^{k} \in T$, then $\left|a_{n}\right| \leq$ $n, n=2,3, \ldots$ For other proofs of this theorem see [4] and [21]. Robertson [17] used Rogosinski's results to show that each function in $T$ can be represented in the form

$$
f(z)=\int_{0}^{\pi} \frac{z}{1-2 z \cos \theta+z^{2}} d \alpha(\theta)
$$

where $a$ is nondecreasing in $[0, \pi]$, and $\alpha(0)=0, \alpha(\pi)=1$.

Received by the editors July 26, 1972 and, in revised form, February 28, 1973. AMS (MOS) subject classifications (1970). Primary 30A40; Secondary $30 \mathrm{~A} 34$.

Key words and phrases. Typically real functions, coefficient bounds, constrained extremal problems.

(1) This paper describes a portion of the author's doctoral thesis written under the direction of Professor Peter L. Duren at the University of Michigan. 
The class $T(c)$ was first studied by Jenkins [12], who found the aomain of variability of $f(z)$ and $f^{\prime}(z)$ when $f \in T(c)$ and $z$ is real. Later Bielecki, Krzyż, and Lewandowski [3] generalized the result for arbitrary $z$; Alenicyn [2] solved the same problem for $f(z)$ by a different method. Using the results of Alenicyn, Goluzina [8] obtained sharp bounds for $|f(z)|, \arg f(z), \operatorname{Re} f(z)$, and $\operatorname{Im} f(z)$ when $f \in T(c)$.

The next result we shall need appears in [3] and [14].

Theorem 1. Let $\Psi$ be real-valued and continuous on $[0, \pi]$. Then the func. tional $\Phi$ defined on (1) by

$$
\Phi(f)=\int_{0}^{\pi} \Psi(\theta) d \alpha(\theta)
$$

assumes its minimum and maximum values in $T(c)$ for a function of the form

$$
f(z)=\frac{c-t}{s-t} \frac{z}{1-s z+z^{2}}+\frac{s-c}{s-t} \frac{z}{1-t z+z^{2}},
$$

where $-2 \leq s \leq c \leq t \leq 2$. If $s=t=c$ we interpret (2) to mean $f(z)=$ $z /\left(1-c z+z^{2}\right)$.

A computation shows that if $f(z)=z+\Sigma_{k=2}^{\infty} a_{k} z^{k} \in T(c)$ is given by (1), then

$$
a_{n}=\int_{0}^{\pi} \frac{\sin n \theta}{\sin \theta} d \alpha(\theta), \quad n=3,4, \ldots
$$

Hence we will study a collection of polynomials that are geometrically similar to the functions $\theta \rightarrow \sin n \theta / \sin \theta$. Our determination of the best upper and lower bounds for $a_{n}, n=3,4, \ldots$, will be divided into four parts:

I. Discussion of the geometrical properties of our collection of polynomials;

II. Solution of the problem when $|c|$ is small;

III. Solution of the problem when $|c|$ is near 2;

IV. Uniqueness of the extremal functions.

Part I. Polynomial geometry. The so-called Chebysher polynomials of the second kind, denoted by $u_{m}(x), m=1,2, \ldots$, satisfy $u_{m}(\theta)=(\sin (m+1) \theta) / \sin \theta$ for each real $\theta$; for several properties of these polynomials, see [22]. We shall deal with a similar collection of monic polynomials.

Definition 2. For each $n, n=1,2, \ldots$, set

$$
r=\left[\frac{n-1}{2}\right], \quad P_{n}(t)=\sum_{k=0}^{r}(-1)^{k}\left(\begin{array}{c}
n-k-1 \\
k
\end{array}\right) t^{n-2 k-1},
$$

where $t$ is real.

Definition 3. Denote by $c_{n}$ the largest critical point of $P_{n}(t), n=1,2, \ldots$. 
The next lemma establishes several useful characteristics of these polynomials.

Lemma 1. The $\left\{P_{n}(t)\right\}_{n=1}^{\infty}$ bave the following properties:

(i) $P_{n}$ is $\left\{\begin{array}{l}\text { even } \\ \text { odd }\end{array}\right\}$ if $n$ is $\left\{\begin{array}{l}\text { odd } \\ \text { even }\end{array}\right\}, n=1,2, \ldots$

(ii) $P_{n}(2 \cos \theta)=(\sin n \theta) / \sin \theta$ for each $\theta \in[-\pi, \pi], n=1,2, \ldots$

(iii) $\sum_{n=1}^{n}\left\{P_{n}(t)\right\} z^{n}=z /\left(1-t z+z^{2}\right)$ for all $z \in D, t \in[-2,2]$.

(iv) If $c$ and $d$ are critical points of $P_{n}(t)$ in $[0, \infty)$ and $c<d$, then $\left|P_{n}(0)\right|$ $<\left|P_{n}(c)\right|<\left|P_{n}(d)\right|<P_{n}(2)=n, n=4,5, \ldots$ $3,4, \ldots$.

(v) $P_{n}\left(c_{n}\right)=\min _{t \in[0,2]} P_{n}(t)$, and $P_{n}$ is concave upward in $\left[c_{n}, \infty\right), n=$

(vi) If $n \geq 4$ is even, then $\left|P_{n}(t)\right|<1 / 2 n|t|$ for all $t \in[-2,2]$. Equality bolds only for $t=0, t= \pm 2$.

Proof. Part (i) is trivial. Next,

$P_{1}(t)=1, P_{2}(t)-t P_{1}(t)=0, P_{n}(t)=t P_{n-1}(t)-P_{n-2}(t), n=3,4, \ldots ;$

hence the identity $\sin n \theta=2 \cos \theta \sin (n-1) \theta-\sin (n-2) \theta$ proves part

(ii) by induction. Note that $\sin n \theta / \sin \theta=\Sigma_{k=0}^{n-1} e^{i(2 k-n+1) \theta}$, hence

(4) $|(\sin n \theta) / \sin \theta|<n$, equality if and only if $\theta=k \pi$ for some integer $k$.

Hence $\sum_{n=1}^{\infty} P_{n}(t) z^{n}$ converges absolutely in $D$ for each fixed $t \in[-2,2]$. Thus $\left(1-t z+z^{2}\right) \sum_{n=1}^{\infty} P_{n}(t) z^{n}=z$, whence part (iii) follows. $P$ arts (iv) and (v) follow from part (ii) and the properties of the functions $\theta \rightarrow(\sin n \theta) / \sin \theta$ [in particular it should be observed that all critical points of $P_{n}$ lie in the open interval $(-2,2)$ ]. Finally, part (vi) is an easy consequence of $(4)$, therefore Lemma 1 is proven.

The constant concavity of $P_{n}$ in $\left[c_{n}, \infty\right)$ shall be used in conjunction with the following geometrical result, which can be easily proven analytically: If $y=f(x)$ is a nonlinear polynomial in a neighborhood of $[a, b]$, and if the line through the points $(a, f(a))$ and $(b, f(b))$ is tangent to $y=f(x)$ at $x=a$, then $f$ cannot have constant concavity in $(a, b)$.

We can now apply Theorem 1 to the constrained coefficient problem. Set

$$
H_{n}(s, t)=\frac{c-t}{s-t} P_{n}(s)+\frac{s-c}{s-t} P_{n}(t), \quad-2 \leq s \leq c \leq t \leq 2,
$$

where $c$ is fixed. We agree to write $H_{n}(c, c)=P_{n}(c)$.

Lemma 2. If $f(z)=z+c z^{2}+\sum_{k=3}^{\infty} a_{k} z^{k} \in T(c)$, then $a_{n}$ satisfies the sharp inequality $\min _{(s, t)} H_{n}(s, t) \leq a_{n} \leq \max _{(s, t)} H_{n}(s, t), n=3,4, \ldots$

Proof. In Theorem 1 , set $\Psi(\theta)=(\sin n \theta) / \sin \theta$; then by (3) we see that the extremal $a_{n}$ occurs for a function of the form (2). However, the $n$th coefficient 
of the function in (2) is clearly $H_{n}(s, t)$, by Lemma 1 . Consequently Lemma 2 follows.

In theory, Lemma 2 allows us to find the exact bounds on $a_{n}$ for each $n$. In practice, however, determination of the minimum and maximum values of $H_{n}(s, t)$ is a nontrivial task. It turns out that when the value $|c|$ is sufficiently small we can solve our problem by exploiting the geometric properties of the polynomials $P_{n}(t)$ in Lemma 1; we can thus avoid working with $H_{n}(s, t)$ in this case. However, when $|c|$ is near 2, we will be forced to appeal to Lemma 2, which necessitates computation of the absolute minimum and maximum of $H_{n}(s, t)$ in the rectangle $-2 \leq s \leq c \leq t \leq 2$.

Part II. Solution for small $|c|$. We turn first to the odd coefficients.

Lemma 3. Let $n \geq 3$ be odd and $f(z)=z+c z^{2}+\sum_{k=3}^{\infty} a_{k} z^{k} \in T(c)$.

(i) We bave $a_{n} \leq n$, with equality if and only if

$$
f(z)=\frac{2+c}{4} \frac{z}{(1-z)^{2}}+\frac{2-c}{4} \frac{z}{(1+z)^{2}}
$$

(ii) If in addition $|c| \leq c_{n}$, then $a_{n} \geq P_{n}\left(c_{n}\right)$. Equality bolds if and only if

$$
f(z)=\frac{c_{n}+c}{2 c_{n}} \frac{z}{1-c_{n} z+z^{2}}+\frac{c_{n}-c}{2 c_{n}} \frac{z}{1+c_{n} z+z^{2}} .
$$

Before proving this lemma we note that (5) can be written as

$$
f(z)=\frac{z\left(1+c z+z^{2}\right)}{\left(1-\dot{c}_{n} z+z^{2}\right)\left(1+c_{n} z+z^{2}\right)} ;
$$

hence if $c_{n}=0$, we interpret (5) to mean that $f(z)=z /\left(1+z^{2}\right)$.

Proof. Choose $a$ so that (1) holds. Then (3) and (4) show that $a_{n} \leq n$ for any function $f \in T$, with equality only for $f(z)=\lambda z /(1-z)^{2}+(1-\lambda) z /(1+z)^{2}$, where $\lambda \in[0,1]$. In our case we must have $a_{2}=2 \lambda-2(1-\lambda)=c$, and part (i) follows. Next, by Lemma $1, a_{n}=\int_{0}^{\pi} P_{n}(2 \cos \theta) d a(\theta) \geq P_{n}\left( \pm c_{n}\right)=P_{n}\left(c_{n}\right)$, with equality only when $a$ is concentrated at $\pm c_{n}$. That is, if $\theta_{n}=\cos ^{-1}\left(c_{n} / 2\right)$, then

$$
\begin{aligned}
\alpha(\theta) & =0 & & \text { if } 0 \leq \theta<\theta_{n}, \\
& =\lambda_{n} & & \text { if } \theta_{n}<\theta<\pi-\theta_{n}, \\
& =1 & & \text { if } \pi-\theta_{n}<\theta \leq \pi,
\end{aligned}
$$

where $\lambda_{n}$ is some constant. The assumption $|c| \leq c_{n}$ guarantees that $0 \leq \lambda_{n} \leq$ 1 , and the function in (5) clearly results. We have now completed the proof of Lemma 3. 
The proof of part (i) can be generalized to show the following result: If $m \geq 2$ is even, $|c| \leq m$, and $f(z)=z+\sum_{k=2}^{\infty} a_{k} z^{k} \in T$ with $a_{m}=c$, then $a_{n} \leq n$, $n=3,5, \ldots$ Equality holds if and only if

$$
f(z)=\frac{m+c}{2 m} \frac{z}{(1-z)^{2}}+\frac{m-c}{2 m} \frac{z}{(1+z)^{2}} .
$$

Hence if $n$ is odd, the maximum value of the $n$th coefficient is not affected by the behavior of any single even coefficient!

The study of even coefficients for fixed $a_{2}$ is more complicated.

Definition 4. If $n \geq 4$ is even, put $F_{n}(t)=\left(P_{n}(t)+n\right) /(t+2), t \in[0,2]$.

By part $(v)$ of Lemma 1 , we are able to conclude that $F_{n}(t)$ attains its minimum in $[0,2]$ at one point only.

Definition 5. We call $r_{n}$ the unique number in $[0,2]$ which satisfies $F_{n}\left(r_{n}\right)$ $=\min _{t \in[0,2]} F_{n}(t)$.

It easily follows that $c_{n}<r_{n}<2$. Furthermore, if we denote by $L$ the collection of all lines tangent to the curve $y=P_{n}(t)$ which pass through the point $(-2,-n)$, then the line through the points $(-2,-n)$ and $\left(r_{n}, P_{n}\left(r_{n}\right)\right)$ is the element of $L$ with minimal slope.

We can now partially solve our problem for even coefficients. In doing so we shall motivate the two definitions above.

Lemma 4. Let $n \geq 4$ be even and $f(z)=z+c z^{2}+\sum_{k=3}^{\infty} a_{k} z^{k} \in T(c)$. Then

$$
(c+2) F_{n}\left(r_{n}\right)-n \leq a_{n} \leq(c-2) F_{n}\left(r_{n}\right)+n .
$$

Equality bolds on the left if and only if $-2 \leq c \leq r_{n}$ and

$$
f(z)=\frac{r_{n}-c}{r_{n}+2} \frac{z}{(1+z)^{2}}+\frac{c+2}{r_{n}+2} \frac{z}{1-r_{n} z+z^{2}},
$$

while equality bolds on the right if and only if $-r_{n} \leq c \leq 2$ and

$$
f(z)=\frac{r_{n}+c}{r_{n}+2} \frac{z}{(1-z)^{2}}+\frac{2-c}{r_{n}+2} \frac{z}{1+r_{n} z+z^{2}}
$$

Proof. Define a function $g$ on $[0,2]$ by $g(t)=P_{n}(t)-F_{n}\left(r_{n}\right) t$. By computing $F_{n}^{\prime}(t)$, we see that $g^{\prime}\left(r_{n}\right)=0$. Furthermore, part $(v)$ of Lemma 1 guarantees that $g^{\prime}(t)$ is increasing in $\left[c_{n}, 2\right)$ and that $\min _{t \in[0,2]} g(t)=\min _{t} \dot{\epsilon}\left[c_{n}, 2\right] d(t)$. Hence by definition of $F_{n}(t)$,

$$
\min _{t \in[0,2]} P_{n}(t)-F_{n}\left(r_{n}\right) t=-n+2 F_{n}\left(r_{n}\right),
$$


and equality holds only for $t=r_{n}$. In particular, we see that $n-2 F_{n}\left(r_{n}\right)>0$; thus part (vi) of Lemma 1 yields

$$
\max _{t \in[0,2]} P_{n}(t)-F_{n}\left(r_{n}\right) t \leq \max _{t \in[0,2]} 1 / 2 n t-F_{n}\left(r_{n}\right) t=n-2 F_{n}\left(r_{n}\right),
$$

with equality only for $t=2$. Combining (6) and (7), we arrive at the inequality

$$
\max _{t \in[0,2]}\left|P_{n}(t)-F_{n}\left(r_{n}\right) t\right| \leq n-2 F_{n}\left(r_{n}\right) .
$$

Now choose $a$ to represent $f$ as in (1). Then

$$
a_{n}=\int_{0}^{\pi}\left(P_{n}(2 \cos \theta)-2 F_{n}\left(r_{n}\right) \cos \theta\right) d a(\theta)+F_{n}\left(r_{n}\right) c
$$

because $f \in T(c)$. Now $P_{n}(t)$ is odd, hence (8) yields

$$
\left|a_{n}-F_{n}\left(r_{n}\right) c\right| \leq n-2 F_{n}\left(r_{n}\right),
$$

which is the desired inequality. The maximum in (8) is assumed only at $t= \pm_{r_{n}}$ and $t= \pm 2$. More explicitly,

$$
\begin{aligned}
& P_{n}\left(-r_{n}\right)+F_{n}\left(r_{n}\right) r_{n}=P_{n}(2)-2 F_{n}\left(r_{n}\right)=n-2 F_{n}\left(r_{n}\right), \\
& P_{n}\left(r_{n}\right)-F_{n}\left(r_{n}\right) r_{n}=P_{n}(-2)+2 F_{n}\left(r_{n}\right)=2 F_{n}\left(r_{n}\right)-n .
\end{aligned}
$$

Thus we concentrate $a$ at $+r_{n}$ and -2 , or at $-r_{n}$ and +2 , to achieve equality on the left side or right side of (9), respectively. The indicated extremal functions are clearly the result (note as in Lemma 3 the restrictions on $c$ are necessary to insure that these functions are actually typically real); hence the proof of Lemma 4 is now finished.

A slight modification of an argument due to Schur [20, pp. 130-132] shows that the sequence $\left\{P_{n}\left(c_{n}\right) / n\right\}_{n=3}^{\infty}$ is strictly increasing, and $\lim _{n \rightarrow \infty} P_{n}\left(c_{n}\right) / n=$ $\cos u_{0}=-0.217 \cdots$, where $u_{0}$ is the unique solution to the equation $u=\tan u$ in $(\pi, 2 \pi)$.

Hence

$$
\lim _{n \rightarrow \infty} \frac{F_{2 n}\left(r_{2 n}\right)}{2 n}=\frac{1+\cos u_{0}}{4}=0.196 \ldots,
$$

which yields an asymptotic estimate for the magnitude of $F_{2 n}\left(r_{2 n}\right)$.

This quantity tan be determined explicitly by digital computer programs, and the following table results. 
Table 1

Numerical values of $F_{n}\left(r_{n}\right)$, correct to three decimal places

$\begin{array}{rrrrrr}n & F_{n}\left(r_{n}\right) & n & F_{n}\left(r_{n}\right) & n & F_{n}\left(r_{n}\right) \\ 2 & 1.000 & 12 & 2.416 & 22 & 4.342 \\ 4 & 1.000 & 14 & 2.797 & 24 & 4.730 \\ 6 & 1.313 & 16 & 3.182 & 26 & 5.119 \\ 8 & 1.668 & 18 & 3.567 & 28 & 5.508 \\ 10 & 2.038 & 20 & 3.954 & 30 & 5.898\end{array}$

Part III. Solution for $|c|$ near 2. We have not solved our problem for two cases: $n$ odd, $|c|>c_{n} ; n$ even, $|c|>r_{n}$. Our aim is to show that in the se cases the only extremal function is $f(z)=z /\left(1-c z+z^{2}\right)=\sum_{k=1}^{\infty} P_{k}(c) z^{k}$. We shall show that $H_{n}(s, t)$ has no absolute minimum in the interior of the rectangle -2 $\leq s \leq c \leq t \leq 2$. The point $s=-2, t=+2$ will then be eliminated as a possible minimum point. All other points on the rectangle's boundary correspond to the function given above.

Our first result will apply to both even and odd coefficients.

Lemma 5. Suppose $n \geq 3, c_{n}<c<2,0<\lambda<1,-2 \leq t_{1}<c<t_{2} \leq 2$, and $P_{n}\left(t_{1}\right) \geq P_{n}\left(c_{n}\right)$. If

$$
g(z)=\lambda \frac{z}{1-t_{1} z+z^{2}}+(1-\lambda) \frac{z}{1-t_{2} z+z^{2}}=z+c z^{2}+\sum_{k=3}^{\infty} b_{k} z^{k}
$$

and

then $b_{n}>M_{n}$.

$$
M_{n}=\min \left\{a_{n} \mid f(z)=z+c z^{2}+\sum_{k=3}^{\infty} a_{k} z^{k} \in T(c)\right\},
$$

Proof. Assume equality holds instead. We easily conclude from parts (iv) and (v) of Lemma 1 that $P_{n}\left(t_{1}\right)<P_{n}\left(t_{2}\right)$. Next we claim that $c_{n} \leq t_{1}<c$. For if $t_{1}<\dot{c}_{n}$, then we could find $s_{1}>0$ so that $P_{n}\left(t_{1}\right) \geq P_{n}\left(t_{1}+s_{1}\right), c_{n} \leq t_{1}+s_{1}<$ $c<t_{2}$ and $\epsilon>0$ so that $(1-\lambda)_{\epsilon}-\lambda s_{1}<0, t_{1}+s_{1}-t_{2}+\epsilon<0, P_{n}\left(t_{2}\right) \geq$ $P_{n}\left(t_{2}-\epsilon\right)>P_{n}\left(t_{1}\right), t_{2}-\epsilon \geq c$. But the $n$th coefficient of the function

$$
f_{1}(z)=\frac{\lambda\left(t_{1}-t_{2}\right)+\epsilon}{t_{1}+s_{1}-t_{2}+\epsilon} \frac{z}{1-\left(t_{1}+s_{1}\right) z+z^{2}}+\frac{s_{1}+(1-\lambda)\left(t_{1}-t_{2}\right)}{t_{1}+s_{1}-t_{2}+\epsilon} \frac{z}{1-\left(t_{2}-\epsilon\right) z+z^{2}}
$$

is smaller than $b_{n}$, a contradiction. Consequently 


$$
c_{n} \leq t_{1}<c<t_{2} \leq 2
$$

as claimed. Now set $b(x)=H_{n}\left(x, t_{2}\right),-2<x<c$. By assumption $x=t_{1}$ is a local minimum of $b$, thus $P_{n}^{\prime}\left(t_{1}^{n}\right)=\left(P_{n}\left(t_{2}\right)-P_{n}\left(t_{1}\right)\right) /\left(t_{2}-t_{1}\right)$. From the remarks following the proof of Lemma 1 , we conclude that $P_{n}(t)$ cannot have constant concavity in the interval $\left(t_{1}, t_{2}\right)$. This fact contradicts (13) and part ( $v$ ) of Lemma 1 , so that the proof is complete.

Corollary 1. If $n \geq 3$ is odd, $c_{n}<c<2,0<\lambda<1,-2 \leq t_{1}<c<t_{2} \leq 2$, and $g(z)$ is given by (11), then $b_{n}>M_{n^{\prime}}$ where $M_{n}$ is as in (12).

Proof. By part (i) of Lemma $1, P_{n}(t)$ is even; hence the hypotheses of Lemma 5 are satisfied.

The case of even coefficients is more difficult because of the complicated nature of $r_{n}$ :

Lemma 6. If $n \geq 4$ is even, $r_{n}<c<2,0<\lambda<1,-2 \leq t_{1}<c<t_{2} \leq 2$, and $g(z)$ and $M_{n}$ are given by (11) and (12), respectively, then $b_{n}>M_{n}$.

Proof. Assume the assertion is false. In view of Lemma 5, we must have $P_{n}\left(t_{1}\right)<P_{n}\left(c_{n}\right)$; hence

$$
t_{1}<-c_{n}
$$

We now consider three cases.

Case I. $t_{1}=-2, t_{2} \leq 2$. The point $r_{n}$ is a local minimum of $F_{n}(t)$, and $P_{n}^{\prime}(t)$ is strictly increasing in $\left(r_{n}, \infty\right)$; hence it easily follows that the $n$th coefficient of

$$
f_{1}(z)=\frac{r_{n}-c}{r_{n}+2} \frac{z}{(1+z)^{2}}+\frac{c+2}{r_{n}+2} \frac{z}{1-r_{n} z+z^{2}} \in T(c)
$$

is smaller than $b_{n}$, a contradiction.

Case II. $t_{1}>-2, t_{2}=2$. By using (14) and Lemma 1 , we see that for an appropriate choice of $\epsilon$, the $n$th coefficient of

$$
f_{2}(z)=\frac{2-c}{2-t_{1}-\epsilon} \frac{z}{1-\left(t_{1}+\epsilon\right) z+z^{2}}+\frac{c-t_{1}-\epsilon}{2-t_{1}-\epsilon} \frac{z}{(1-z)^{2}}
$$

will be smaller than $b_{n}$, another contradiction.

Case III. $t_{1}>-2, t_{2}<2$. The point $\left(t_{1}, t_{2}\right)$ is a relative minimum of $H_{n}$; thus $P_{n}^{\prime}\left(t_{1}\right)=\left(P_{n}\left(t_{2}\right)-P_{n}\left(t_{1}\right)\right) /\left(t_{2}-t_{1}\right)=P_{n}^{\prime}\left(t_{2}\right)$. Hence we must have $t_{2}=$ $-t_{1}$. We employ part (vi) of Lemma 1 to deduce that

$$
P_{n}^{\prime}\left(t_{2}\right)=\frac{P_{n}\left(t_{2}\right)}{t_{2}} \leq \frac{P_{n}\left(r_{n}\right)+n}{r_{n}+2}=P_{n}^{\prime}\left(r_{n}\right) \text {. }
$$


This result is a contradiction, since $t_{2}>c>r_{n}$, whereas $P_{n}^{\prime}(t)$ is strictly increasing in $\left(r_{n}, \infty\right)$. The proof is now complete.

We can now finish solving our problem, except for the identification of all extremal functions.

Lemma 7. Let $f(z)=z+c z^{2}+\sum_{k=3}^{\infty} a_{k} z^{k} \in T(c)$ be represented by $a$ in (1). If $n$ is odd and $|c|>\left|c_{n}\right|$, then $a_{n} \geq P_{n}(c)$. If $n$ is even, then we bave $a_{n} \geq$ $P_{n}(c)$ if $c>r_{n^{\prime}} a_{n} \leq P_{n}(c)$ if $c<-r_{n}$. If $a$ is a step function, then equality bolds in any of the three inequalities above if and only if $f(z)=z /\left(1-c z+z^{2}\right)$.

Proof. First suppose

$$
\begin{array}{cl}
c>c_{n} & \text { if } n \text { is odd, } \\
>r_{n} & \text { if } n \text { is even. }
\end{array}
$$

By Corollary 1 and Lemma 6 , the absolute minimum of $H_{n}(s, t)$ is not assumed when $-2 \leq s<c<t \leq 2$. Since $H_{n}(c, t)=H_{n}(s, c)=P_{n}^{n}(c)$ for all $s$ and $t$, the inequality $a_{n} \geq P_{n}(c)$ follows from Lemma 2 .

Next assume $\alpha$ is a step function. If $\alpha$ has at most two discontinuities, then we can have $a_{n}=P_{n}(c)$ if and only if $f(z)=z /\left(1-c z+z^{2}\right)$. If $\alpha$ has more than two discontinuities, we write $A_{-1}=0$ and

$$
\begin{aligned}
\alpha(\theta) & =A_{0}=0 & & \text { if } 0 \leq \theta \leq \theta_{1}, \\
& =A_{k} & & \text { if } \theta_{k}<\theta<\theta_{k+1}, k=1, \ldots, m-1, \\
& =A_{m}=1 & & \text { if } \theta_{m} \leq \theta \leq \pi .
\end{aligned}
$$

By setting $A_{l-1}=A_{l}$ if necessary, we can assume that the number $\theta_{l}=\cos ^{-1}(c / 2)$ occurs among the $\theta_{k}, k=1, \cdots, m$. Then

$$
f(z)=\left(A_{l}-A_{l-1}\right) z /\left(1-c z+z^{2}\right)+\left(1-A_{l}+A_{l-1}\right) b(z)
$$

where

$$
b(z)=\int_{0}^{\pi} \frac{z}{1-2 z \cos \theta+z^{2}} d \beta(\theta) \in T(c)
$$

and $\beta$ has no discontinuity at $\theta_{l}$. It is now possible (see [14, Theorem 1]) to find constants $d_{1}, \cdots, d_{m-2} \geq 0$ and nondecreasing step functions $\beta_{1}, \cdots, \beta_{m-2}$ such that

$$
\sum_{k=1}^{m-2} d_{k}=1, \quad \int_{0}^{\pi} \cos \theta d \beta_{k}(\theta)=c / 2, \quad k=1, \ldots, m-2,
$$

and 


$$
\beta(\theta)=\sum_{k=1}^{m-2} d_{k} \beta_{k}(\theta)
$$

for all but finitely many $\theta$ in $[0, \pi]$; each $\beta_{k}$ has at most two discontinuities. If $a_{n}=P_{n}(c)$, then the $n$th coefficient of $b$ must also be $P_{n}(c)$; hence $b(z)=$ $z /\left(1-c z+z^{2}\right)=f(z)$.

We have now completely proven the lemma for $c>0$. If $\dot{c}<0$, we set $g(z)=$ $-f(-z)$ and apply what we have just shown to $g$. The desired inequalities follow from part (i) of Lemma 1.

Part IV. Uniqueness of extremal functions. We now state our complete solution to the constrained coefficient problem.

Theorem 2. Suppose $f(z)=z+c z^{2}+\sum_{k=3}^{\infty} a_{k} z^{k} \in T(c)$.

1. If $n \geq 3$ is odd, then

$$
\begin{array}{cc}
P_{n}\left(c_{n}\right) \leq a_{n} \leq n & \text { if }|c| \leq c_{n}, \\
P_{n}(c) \leq a_{n} \leq n & \text { if }|c| \geq c_{n} .
\end{array}
$$

2. If $n \geq 4$ is even, then

$$
\begin{aligned}
(c+2) F_{n}\left(r_{n}\right)-n & \leq a_{n} \leq P_{n}(c) & & \text { if }-2 \leq c \leq-r_{n}, \\
(c+2) F_{n}\left(r_{n}\right)-n & \leq a_{n} \leq(c-2) F_{n}\left(r_{n}\right)+n & & \text { if }|c| \leq r_{n}, \\
P_{n}(c) & \leq a_{n} \leq(c-2) F_{n}\left(r_{n}\right)+n & & \text { if } r_{n} \leq c \leq 2 .
\end{aligned}
$$

Equality bolds on the left-band sides only for

$$
\begin{aligned}
& f(z)=\frac{c_{n}+c}{2 c_{n}} \frac{z}{1-c_{n} z+z^{2}} \frac{c_{n}-c}{2 c_{n}} \frac{z}{1+c_{n} z+z^{2}} \text { in (15); } \\
& f(z)=\frac{r_{n}-c}{r_{n}+2} \frac{z}{(1+z)^{2}}+\frac{c+2}{r_{n}+21-r_{n} z+z^{2}} \text { in (17), (18); } \\
& f(z)=\frac{z}{1-c z+z^{2}} \text { in (16), (19). }
\end{aligned}
$$

Equality bolds on the right-band sides only for

$$
\begin{aligned}
& f(z)=\frac{2+c}{4} \frac{z}{(1-z)^{2}}+\frac{2-c}{4} \frac{z}{(1+z)^{2}} \text { in (15), (16); } \\
& f(z)=\frac{z}{1-c z+z^{2}} \text { in (17); } \\
& f(z)=\frac{r_{n}+c}{r_{n}+2} \frac{z}{(1-z)^{2}}+\frac{2-c}{r_{n}+2} \frac{z}{1+r_{n}^{\cdot} z+z^{2}} \text { in (18), (19). }
\end{aligned}
$$


Proof. In view of Lemmas 3, 4, and 7, we need only show that if $f(z)=z+$ $c z^{2}+\sum_{k=3}^{\infty} a_{k} z^{k}$ is extremal for our problem, and $a$ represents $f$ as in (1), then $\alpha$ must be a step function. To do this, let $\theta_{1}, \ldots, \theta$, be all the zeros of $((\sin n \theta) / \sin \theta)^{\prime}$ in $(0, \pi)$, where $0=\theta_{0}<\theta_{1}<\ldots<\theta_{r}<\theta_{r+1}=\pi$. Suppose there exists a point $\theta^{\prime}$ in some interval $\left(\theta_{l}, \theta_{l+1}\right)$ such that $a$ is not constant in any neighborhood of $\theta^{\prime}$. Using the variational method of Pfaltźgraff and Pinchuk [16, Theorem 4.2], we obtain a function $f_{*} \in T(c)$ of the form

$$
f_{*}(z)=f(z)-\epsilon \int_{e}\left[\frac{2 z^{2} \sin \theta}{\left(1-2 z \cos \theta+z^{2}\right)^{2}}+\lambda \sin \theta\right]|\alpha(\theta)-x| d \theta+O\left(\epsilon^{2}\right),
$$

where $e \subseteq\left(\theta_{l}, \theta_{l+1}\right)$ is a closed interval about $\theta^{\prime}, \lambda$ and $x$ are constants, and the error term $O\left(\epsilon^{2}\right)$ is uniform on compact subsets of the unit disk. The quantity $\epsilon$ can be positive or negative, provided $|\epsilon|$ is sufficiently small. The $n$th coefficient $a_{n *}$ of $f_{*}$ is given by

$$
a_{n *}=a_{n}+\epsilon \int_{e}\left(\frac{\sin n \theta}{\sin \theta}\right)^{\prime}|\alpha(\theta)-x| d \theta+O\left(\epsilon^{2}\right)
$$

hence we conclude that $\int_{e}((\sin n \theta) / \sin \theta)^{\prime}|a(\theta)-x| d \theta=0$, a contradiction. Consequently, $\alpha$ is constant on $\left(\theta_{k}, \theta_{k+1}\right), k=0, \ldots, r$, and the proof is complete.

It should be pointed out that the variational method was used only to show the uniqueness of the extremal function in the case $a_{n}=P_{n}(c), f(z)=$ $z /\left(1-c z+z^{2}\right)$. The rest of the problem was solved on the elementary level.

Theorem 2 yields the following result on odd typically real functions: if $f(z)=z+\Sigma_{n=1}^{\infty} a_{2 n+1} z^{2 n+1} \in T$, then $P_{2 n+1}\left(c_{2 n+1}\right) \leq a_{2 n+1} \leq 2 n+1, n=1,2$, .... Equality holds on the left or right side only for

$$
f(z)=\frac{z\left(1+z^{2}\right)}{\left(1+z^{2}\right)^{2}-c_{n} z^{2}} \text { or } f(z)=z \frac{1+z^{2}}{\left(1-z^{2}\right)^{2}},
$$

respectively. This assertion of course holds under the weaker hypothesis that $f^{\prime \prime}(0)=0$. (A similar phenomenon occurs in the class $S^{*}$ of normalized starlike univalent functions: Goluzin [S] shows that if $f(z)=z+\Sigma_{k=2}^{\infty} a_{k} z^{k} \in S^{*}$ then $\left|a_{k}\right| \leq 1, k=3,5,7, \ldots$, if $f$ is odd. Later [6] he shows that $\left|a_{k}\right| \leq 1, k=3$, $4,5, \ldots$, provided only $a_{2}=0$.)

If $f(z)=z+c z^{2}+a_{3} z^{3}+\cdots \epsilon T(c)$, then $c^{2}-1 \leq a_{3} \leq 3$. The left-hand side easily follows from the Schwarz inequality, but tracing the cases of equality is cumbersome. Note also that if we represent $f$ by $(1)$ and set $\beta(t)=$ $a\left[\cos ^{-1}(-t / 2)\right]$, then

$$
-a_{2}=\int_{-2}^{2} t d \beta(t)=-c, \quad a_{3}+1-c^{2}=\int_{-2}^{2} t^{2} d \beta(t)-c^{2} .
$$


Thus finding the best bounds on $a_{3}$ amounts to minimizing and maximizing the variance of a mass (in fact, probability) distribution when the mean is given. No such interpretation appears possible for higher coefficients $a_{n}, n \geq 4$.

This work represents part of the author's doctoral dissertation written at the University of Michigan. I would like to thank my thesis advisor Professor Peter L. Duren for his assistance in this project, my wife Virginia for her writing of the computer program that produced Table 1, and the referee for several helpful comments.

\section{REFERENCES}

1. L. V. Ahlfors et al., Analytic functions, Princeton Univ. Press, Princeton, N. J., 1960.

2. Ju. E. Alenicyn, On the ranges of systems of coefficients of functions representable as a sum of Stieltjes integrals, Vestnik Leningrad Univ. 17 (1962), no. 7, 25-41. (Russian) MR 25 \#2179.

3. A. Bielecki, J. Krzyż and Z. Lewandowski, On typically-real functions with a preassigned second coefficient, Bull. Acad. Polon. Sci. Sér. Sci. Math. Astronom. Phys. 10 (1962), 205-208. MR 25 \#2182.

4. J. Dieudonné, Sur les fonctions univalentes, C. R. Acad. Sci. Paris Sér. A-B 192 (1931), 1148-1150.

5. G. M. Goluzin, On some estimates for functions which map the circle conformally and univalently, Mat. Sb. 36 (1929), 152-172. (Russian).

6. - On certain coefficient estimates for schlicht functions, Mat. Sb. 3 (1938), 321-330. (Russian).

7. $\longrightarrow$ On the theory of univalent functions, Mat. Sb. 28 (70)(1951), 351-358. (Russian) MR 13, 639.

8. E. G. Goluzina, On typically-real functions with fixed second coefficient, Vestnik Leningrad Univ. 17 (1962), no. 7, 62-70. (Russian) MR 25 \#2180.

9. T. H. Gronwall, Sur la déformation dans la représentation conforme sous des conditions restrictives, C. R. Acad. Sci. Paris Sér A-B 162 (1916), 316-318.

10. $\longrightarrow$ On the distortion in conformal mapping when the second coefficient in the mapping function has an assigned value, Nat. Acad. Proc. 6 (1920), 300-302.

11. J. A. Jenkins, On a problem of Gronwall, Ann. of Math. (2) 59 (1954), 490-504. MR 15, 786.

12. - Some problems for typically real functions, Canad. J. Math. 13 (1961), 299-304. MR 22 \#12227.

13. N. A. Lebedev and I. M. Milin, On the coefficients of certain classes of analytic functions, Mat. Sb. 28 (70) (1951), 359-400. (Russian) MR 13, 640.

14. G. B. Leeman, Constrained extremal problems for families of Stieltjes integrals, Arch. Rational Mech. Anal. (to appear).

15. R. Nevanlinna, Ober die konforme Abbildung von Sternegebieten, Finska Vetenskaps-Soc. Förh. $63(1920 / 21)$, Sect. A, no. 6.

16. J. Pfaltzgraff and B. Pinchuk, A variational method for classes of meromorphic functions, J. Analyse Math. 24 (1971), 101-150. MR 43 \#613. 
17. M. S. Robertson, On the coefficients of a typically-real function, Bull. Amer. Math. Soc. 41 (1935), 565-572.

18. W. Rogosinski, Ober positive harmonische Sinusentwicklungen, Jber. Deutsch. Math.-Verein. 40 (1931), 33-35.

19. - Ober positive harmonische Entwicklungen und typisch-reelle Potenzreihen, Math. Z. 35 (1932), 93-121.

20. 1. Schur, Ûber die Koeffizientensummen einer Potenzreihe mit positivem reellem Teil, Arch. Math. Phys. 27 (1918), 126-135.

21. O. Szász, Ober Funktionen die den Einheitskreis schlicht abbilden, Jber. Deutsch. Math.-Verein. 42 (1932), 73-75.

22. G. Szegö, Orthogonal polynomials, Amer. Math. Soc. Colloq. Publ., vol. 23, Amer. Math. Soc., Providence, R. I., 1939. MR 1, 14.

IBM THOMAS J. WATSON RESEARCH CENTER, P.O. BOX 218, YORKTOWN HEIGHTS, NEW YORK 10598 